Pacific Journal of Mathematics

RINGS WITH QUIVERS THAT ARE TREES

alpo Fuller and Joel K. mAc 


\title{
RINGS WITH QUIVERS THAT ARE TREES
}

\author{
K. R. Fuller and Joel HaACK
}

\begin{abstract}
Associated with each artinian ring $R$ are two diagrams called the left and right quivers of $R$. We generalize a well-known theorem on hereditary serial rings by proving that if these quivers have no closed paths then $R$ is a factor ring of a certain ring of matrices over a division ring. It follows that the categories of finitely generated left and right $R$-modules are Morita dual to one another. Applying our theorem and theorems of Gabriel and Dlab and Ringel, we show how to write explicit matrix representations of all hereditary algebras of finite module type.
\end{abstract}

A quiver is, in the terminology of Gabriel [8], [9], a finite set of points (vertices) connected by arrows. Given an artinian ring $R$ and a basic set of primitive idempotents $e_{1}, \cdots, e_{n}$ of $R$ (see, for example, [1, §27]), one forms $\mathscr{Q}\left({ }_{R} R\right)$ the left quiver of $R$ (see [11]): The vertices of $\mathscr{Q}\left({ }_{R} R\right)$ are $v_{1}, \cdots, v_{n}$, one for each basic idempotent, with $n_{i j}$ arrows from $v_{i}$ to $v_{j}$ iff $R e_{j} / J e_{j}$ appears exactly $n_{i j}$ times in a direct decomposition of the semisimple left $R$-module $J e_{i} / J^{2} e_{i}$. The right quiver $\mathscr{Q}\left(R_{R}\right)$ is formed similarly, with vertices $v_{1}^{\prime}, \cdots, v_{n}^{\prime}$ and $n_{i j}^{\prime}$ arrows from $v_{i}^{\prime}$ to $v_{j}^{\prime}$ iff $e_{j} R / e_{j} J$ appears exactly $n_{i j}^{\prime}$ times in a direct decomposition of $e_{i} J / e_{i} J^{2}$. Note that $n_{i j}^{\prime} \neq 0$ iff $n_{j i} \neq 0$. Also, $R$ is indecomposable iff $\mathscr{Q}\left({ }_{R} R\right)$ is connected, i.e., there is a nonoriented path from $v_{i}$ to $v_{j}$ for every $i, j=1, \cdots, n$.

A quiver $Q$ is called a tree in case it is connected and contains no cycles, i.e., in case it has a unique nonoriented path from $v_{i}$ to $v_{j}$, for every $i, j$. Let $\mathscr{Q}$ be such a quiver. Then the vertices of $Q$ are partially ordered by $\leqq$, where $v_{i} \leqq v_{j}$ iff there is an oriented path from $v_{j}$ to $v_{i}$ (or $i=j$ ), and we can relabel the vertices so that $v_{i} \$ v_{j}$ implies $i \leqq j$. Having done this, we see that for any ring $D$, the set of matrices

$$
T=\left\{\llbracket d_{i j} \rrbracket \mid d_{i j} \in D, d_{i j}=0 \quad \text { if } \quad v_{i} \not v_{j}\right\}
$$

is a subring of the ring of upper triangular matrices over $D$. Moreever, if $D$ is a division ring, then $\mathscr{Q}\left({ }_{T} T\right)=\mathscr{Q}, \mathbb{Q}\left(T_{T}\right)$ is the dual quiver of $\mathscr{Q}$, and $T$ is the unique basic tic tac toe ring (in the sense of Mitchell $[12, \S 10.8]$ ) over $D$ with left quiver $\mathscr{Q}$.

Murase [14] showed that an indecomposable artinian ring whose quivers are of the form

$$
v_{1} \longleftarrow v_{2} \longleftarrow v_{3} \cdots v_{n-1} \longleftarrow v_{n}
$$


is a factor ring of a block upper triangular matrix ring (i.e., of one whose basic ring is an upper triangular matrix ring) over a division ring. (Goldie [10] proved a similar result.) A ring with such a quiver is a serial ring, and an indecomposable hereditary artinian ring is serial iff it has quivers of this form. We extend this result, showing that any artinian ring whose quivers are trees is a factor ring of a tic tac toe ring over a division ring. As an application we also prove that such rings are self-dual in the sense that there is a Morita duality between their categories of finitely generated left and right modules.

Before proceeding to the proofs we note that, by the work of Gabriel [8], ]9], and Dlab and Ringel [4], an indecomposable hereditary algebra over an algebraically closed field is of finite module type iff its quivers are Dynkin diagrams of type $A_{n}, D_{n}, E_{6}, E_{7}$, or $E_{8}$. These diagrams are all trees, so the theorem we are about to prove allows one to apply Gabriel's argument [8] (see also [2], [11]) to show that any artinian ring with quivers of type $A_{n}, D_{n}, E_{6}, E_{7}$, or $E_{8}$ is a ring of finite module type.

LEMMA 1. Let $R$ be an artinian ring with $e_{1}, \cdots, e_{n}$ a basic set of primitive idempotents. If $R e_{i} / J e_{i}$ is isomorphic to a direct summand of $J^{k} e_{j} / J^{k+1} e_{j}$, then in $\mathscr{Q}\left({ }_{R} R\right)$ there is an oriented path from $v_{j}$ to $v_{i}$ of length $k$. Moreover, if in addition $R$ is hereditary, then the converse is also true.

Proof. We induct on $k$. The cases $k=0$ and $k=1$ follow immediately from the definition of a quiver. Now let $R e_{i} / J e_{i}$ be isomorphic to a direct summand of $J^{k} e_{j} / J^{k+1} e_{j}$. Let

$$
\bigoplus_{r=1}^{t} R e_{j_{r}} \stackrel{f}{\longrightarrow} J^{k-1} e_{j} \longrightarrow 0
$$

be a projective cover. Then $f$ induces an epimorphism

$$
\bigoplus_{r=1}^{t}\left(J e_{j_{r}} / J^{2} e_{j_{r}}\right) \stackrel{\bar{f}}{\longrightarrow} J^{k} e_{j} / J^{k+1} e_{j} \longrightarrow 0 \text {. }
$$

Since $\bigoplus_{r=1}^{t}\left(J e_{j_{r}} / J^{2} e_{j_{r}}\right)$ and $J^{k} e_{j} / J^{k+1} e_{j}$ are semisimple $R / J$-modules, $\bar{f}$ splits. Thus there exists an $r$ with $R e_{i} / J e_{i}$ isomorphic to a direct summand of $J e_{j_{r}} / J^{2} e_{j_{r}}$. By induction, there is an oriented path of length $(k-1)$ from $v_{j}$ to $v_{j_{r}}$ and one of length 1 from $v_{j_{r}}$ to $v_{i}$, which combine to give the desired path of length $k$.

For the moreover part, suppose we have an oriented path

$$
v_{i}=v_{i_{k}} \longleftarrow v_{i_{k-1}} \cdots v_{i_{1}} \longleftarrow v_{i_{0}}=v_{j} \text {. }
$$

Assume that $R e_{i_{m}} / J e_{i_{m}}$ is a direct summand of $J^{m} e_{j} / J^{m+1} e_{j}(m<k)$. 
Then since $R$ is hereditary, $J^{m} e_{j} \cong R e_{i_{m}} \oplus M$, some $M$. Thus

$$
J^{m+1} e_{j} / J^{m+2} e_{j} \cong J e_{i_{m}} / J^{2} e_{i_{m}} \oplus J M / J^{2} M,
$$

and we are done since $R e_{i_{m+1}} / J e_{i_{m+1}}$ is a direct summand of $J e_{i_{m}} / J^{2} e_{i_{m}}$. Now we are ready to prove the promised result.

THEOREM 2. If the left and right quivers of an artinian ring $R$ are trees, then there is an indecomposable tic tac toe ring $T$ over a division ring $D$ such that $R$ is isomorphic to a factor ring of $T$. Moreover, $\mathbb{Q}\left({ }_{R} R\right)=\mathbb{Q}\left({ }_{T} T\right)$; and $R$ is hereditary iff $R \cong T$.

Proof. It is easy to see that a ring is Morita equivalent to an upper triangular tic tac toe ring over a division ring $D$ iff it is isomorphic to a (block-upper-triangular) tic tac toe ring over $D$. Thus we may assume that $R$ is basic.

Suppose that $\mathbb{Q}=\mathbb{Q}\left({ }_{R} R\right)$ and $\mathscr{Q}\left(R_{R}\right)$ are trees, and correspondingly, relabel the vertices of $Q$ and the idempotents of $R$ as in the earlier discussion. In particular then, $v_{1}$ is minimal with respect to the partial order $\leqq$, and hence no arrows leave $v_{1}$.

Note that for each basic idempotent $e_{i}, e_{i} R e_{i}$ is a division ring since $e_{i} J e_{i}=0$ by Lemma 1 . For each pair of idempotents $e_{p}$ and $e_{q}$ with an arrow from $v_{q}$ to $v_{p}$ in $Q$ (and hence one arrow from $v_{p}^{\prime}$ to $v_{q}^{\prime}$ in $\mathscr{Q}\left(R_{R}\right)$ ), we have a left $e_{p} R e_{p}$ - right $e_{q} R e_{q}$-bimodule $e_{p} J e_{q}$ with $\operatorname{dim}\left(e_{e_{p} R e_{p}} e_{p} J e_{q}\right)=1=\operatorname{dim}\left(e_{p} J e_{q e_{q} R e_{q}}\right)$. So we may choose $e_{p q} \in e_{p} J e_{q}$ with $e_{p q} \neq 0$ and define a division ring isomorphism $\sigma_{p q}: e_{p} R e_{p} \rightarrow e_{q} R e_{q}$ via $x e_{p q}=e_{p q} \sigma_{p q}(x)$ for $x \in e_{p} R e_{p}$. Since $\mathscr{Q}$ is connected, we have $e_{p} R e_{p} \cong e_{r} R e_{r}$ for all primitive idempotents $e_{p}$ and $e_{r}$. Define $e_{i i}=e_{i}$ and for each $v_{i} \leqq v_{j}$ with oriented path

$$
v_{i}=v_{i_{0}} \longleftarrow v_{i_{1}} \cdots v_{i_{k-1}} \longleftarrow v_{i_{k}}=v_{j},
$$

define

$$
e_{i j}=e_{i} e_{i_{0} i_{1}} \cdots e_{i_{k-1} i_{k}} e_{j} \text {. }
$$

For $v_{p} \leftarrow v_{q}$ in $\mathscr{Q}$, define $\gamma_{p q}=\sigma_{p q}$ and $\gamma_{q p}=\sigma_{p q}^{-1}$. Now let $v_{1}=$ $v_{i_{0}}, v_{i_{1}}, \cdots, v_{i_{k}}=v_{j}$ be the vertices of a nonoriented path from $v_{1}$ to $v_{j}$ for $j \neq 1$. Define

Define $\sigma_{11}=1_{e_{1} R e_{1}}$. Let

$$
\sigma_{1 j}=\gamma_{i_{k-1} i_{k}} \circ \cdots \circ \gamma_{i_{1} i_{2}} \circ \sigma_{i_{0} i_{1}} \quad \text { for } \quad j=2, \cdots, n \text {. }
$$

$$
D=\left\{\sum_{j=1}^{n} \sigma_{1 j}(x) \mid x \in e_{1} R e_{1}\right\} .
$$

Then $D \cong e_{j} R e_{j}$ and $D$ is a division subring of $R$.

Let $v_{i} \leqq v_{j}$ via an oriented path of length $k$. Then $e_{i} R e_{j}=e_{i} J^{k} e_{j}$ 
by Lemma 1. It is then straightforward to verify that $D e_{i j}=$ $e_{i} J^{k} e_{j}$, using the equalities $D e_{p}=e_{p} R e_{p}$ and $e_{p} R e_{p q}=e_{p} R e_{p} e_{p q}=e_{p} J e_{q}$ for $v_{p} \leftarrow v_{q}$. Hence we have shown that

$$
R=\sum_{v_{i} \leq v_{j}} D e_{i j}
$$

Next we claim that $d e_{i j}=e_{i j} d$ for any $d \in D$ and $v_{i} \leqq v_{j}$ in $\mathscr{Q}$. Suppose we have $v_{p} \leftarrow v_{q}$. Let $v_{1}=v_{i_{0}}, v_{i_{1}}, \cdots, v_{i_{m}}=v_{q}$ be the nonoriented path from $v_{1}$ to $v_{q}$. If $v_{p}=v_{i_{m-1}}$, then $\sigma_{1 q}=\sigma_{p q} \circ \sigma_{1 p}$, and

$$
\begin{aligned}
e_{p q}\left(\sum_{r=1}^{n} \sigma_{1 r}(x)\right) & =e_{p q} \sigma_{1 q}(x)=e_{p q} \sigma_{p q}\left(\sigma_{1 p}(x)\right)=\sigma_{1 p}(x) e_{p q} \\
& =\left(\sum_{r=1}^{n} \sigma_{1 r}(x)\right) e_{p q} .
\end{aligned}
$$

If $v_{p} \neq v_{i_{m-1}}$, then $\sigma_{1 p}=\sigma_{p q}^{-1} \circ \sigma_{1 q}$, and

$$
\begin{aligned}
e_{p q}\left(\sum_{r=1}^{n} \sigma_{1 r}(x)\right) & =e_{p q} \sigma_{1 q}(x)=\sigma_{p q}^{-1}\left(\sigma_{1 p}(x)\right) e_{p q}=\sigma_{1 p}(x) e_{p q} \\
& =\left(\sum_{r=1}^{n} \sigma_{1 r}(x)\right) e_{p q} .
\end{aligned}
$$

Now the claim follows by induction on the length of the path from $v_{j}$ to $v_{i}$.

Let $T$ be the tic tac toe ring

$$
T=\left\{\llbracket d_{i j} \rrbracket \mid d_{i j} \in D, d_{i j}=0 \quad \text { if } \quad v_{i} \not \leq v_{j}\right\} .
$$

Define

$$
\Phi: T \longrightarrow R \quad \text { via } \Phi: \llbracket d_{i j} \rrbracket \longmapsto \sum_{v_{i} \leq v_{j}} d_{i j} e_{i j} \cdot
$$

Since the elements of $D$ commute with each $e_{i j}$, and since

$$
e_{k m} e_{p q}=\left\{\begin{array}{lll}
e_{k q} & \text { if } \quad m=p \\
0 & \text { if } \quad m \neq p
\end{array}\right.
$$

$\Phi$ is a ring homomorphism. Also $\Phi$ is onto by (*).

Clearly $\mathscr{Q}\left({ }_{T} T\right)=\mathscr{Q}\left({ }_{R} R\right)$. If $R$ is hereditary, then for $v_{i} \leqq v_{j}$ with oriented path of length $k, D e_{i j}=e_{i} J^{k} e_{j} \neq 0$ by Lemma 1. So $e_{i j} \neq 0$ and $\Phi$ is an isomorphism. If $T$ is a tic tac toe ring whose quivers are trees, then $T$ is hereditary by [12, Theorem IX. 10.9].

One could apparently use an argument similar to the one in [4, Proposition 10.2] to show that the rings of Theorem 2 are factor rings of so-called tensor rings (see [5]). The same argument, however, shows that rings with quivers 


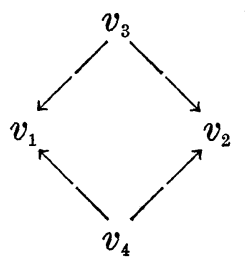

and

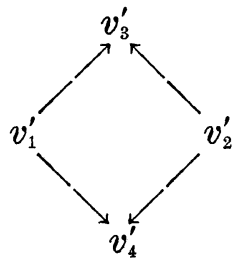

are also tensor rings. But these need not be tic tac toe rings. Indeed, let $\varphi$ be an automorphism of a division ring $D$ which does not fix the center of $D$. Then the ring $R_{\varphi}$ of matrices

$$
\left(\begin{array}{cccc}
a & 0 & x & m \\
& b & y & z \\
& & c & 0 \\
& & & d
\end{array}\right)
$$

with all entries in $D$ except $m \in{ }_{D} M_{D}$ where ${ }_{D} M={ }_{D} D$ and multiplication in $M_{D}$ is given by $m \cdot d=m \varphi(d)$, is a tensor ring that is not a tic tac toe ring. In contrast, by Theorem 2 or originally by Murase in [14], the ring $S_{\varphi}$ of matrices

$$
\left(\begin{array}{cc}
a & m \\
0 & b
\end{array}\right)
$$

with $a, b \in D$ and $m \in M$ is isomorphic to the ring of upper triangular $2 \times 2$ matrices over $D$. (A word of caution: Associativity is lost if one tries this trick for $3 \times 3$ upper triangular matrices.) The ring $R_{\varphi}$ fails to behave similarly, for the center of $R_{\varphi}$ is all scalar matrices $c I$ with $\varphi(c)=c \in \operatorname{center}(D)$.

Note also that the above example indicates that Theorem 2 does not extend to include rings whose quivers are not trees.

A gap in the Morita duality theory that begs to be filled is the nearly total lack of knowledge of which artinian rings (in addition to artin algebras and $Q F$ rings) are self-dual. The characterization of artinian rings whose quivers are trees given in Theorem 2 enables us to show that such rings are self-dual. We employ the following lemma whose proof is dual to that of [7, Lemma 4]. In what follows, $E(M)$ is the injective envelope of $M$ and $\operatorname{Soc}_{k}(M)$ is the $k$ th term in the lower Loewy series of $M$.

LEMma 3. Let $R$ be any ring. Then the following statements about a left $R$-module $M$ are equivalent:

(a) $M$ is distributive.

(b) For each simple left $R$-module $T$, the set of submodules $\left\{\operatorname{ker} \gamma \mid \gamma \in \operatorname{Hom}_{R}(M, E(T))\right\}$ is linearly ordered. 
(c) For each simple left $R$-module $T$ the right $\operatorname{End}\left({ }_{R} E(T)\right)$ module $\operatorname{Hom}_{R}(M, E(T))$ is uniserial.

Proposition 4. If $R$ is an artinian ring whose quivers are trees, then there is a Morita duality between the categories of finitely generated left and finitely generated right $R$-modules.

Proof. Assume that $R$ is indecomposable and basic with identity element a sum of orthogonal primitive idempotents $1_{R}=e_{1}+\cdots+e_{n}$. Let $E_{i}=E\left(R e_{i} / J e_{i}\right)$ for $i=1, \cdots, n$, let $E=E_{1} \oplus \cdots \oplus E_{n}$, and let $S=\operatorname{End}\left({ }_{R} E\right)$. Then ()$^{*}=\operatorname{Hom}_{R}\left({ }_{-, R} E_{S}\right)$ defines a duality between the categories of finitely generated left $R$-modules and finitely generated right $S$-modules [13] and [7, Lemma 5]. Write $1_{S}=f_{1}+\cdots+f_{n}$ where the $f_{i}$ are the orthogonal primitive idempotents in $S$ such that $E f_{i}=E_{i}$. Let $N=J(S)$. We will show that the quivers of $S$ are the same as the quivers of $R$.

From the results in $[1, \S 24]$, we see that for $i=1, \cdots, n$,

$$
\begin{aligned}
& \left(R e_{i} / J e_{i}\right)^{*} \cong\left(\operatorname{Soc} E_{i}\right)^{*} \cong f_{i} S / f_{i} N \\
& \left(\operatorname{Soc}_{2}\left(E_{i}\right) / \operatorname{Soc}\left(E_{i}\right)\right)^{*} \cong f_{i} N / f_{i} N^{2} .
\end{aligned}
$$

So by [7, Lemma 5], $f_{i} N / f_{i} N^{2}$ is square free, and by [6, Theorem 2.4],

$$
e_{i} R / e_{i} J \text { embeds in } e_{j} J / e_{j} J^{2}
$$

iff $R e_{i} / J e_{i}$ embeds in $\operatorname{Soc}_{2}\left(E_{j}\right) / \operatorname{Soc}\left(E_{j}\right)$

iff $f_{i} S / f_{i} N \cong\left(R e_{i} / J e_{i}\right)^{*}$ embeds in $\left(\operatorname{Soc}_{2}\left(E_{j}\right) / \operatorname{Soc}\left(E_{j}\right)\right)^{*}$

$$
\cong f_{j} N / f_{j} N^{2} \text {. }
$$

Thus the right quiver of $S$ is the same as the right quiver of $R$.

Now to see that the left quivers of $R$ and $S$ are the same we need only show that $\operatorname{dim}\left({ }_{f_{i} S f_{i}} f_{i} S f_{j}\right)=0$ or 1 for all $i, j$. But (writing maps on the right), $f_{i} S f_{j} \cong \operatorname{Hom}_{R}\left(E_{i}, E_{j}\right) \cong \operatorname{Hom}_{e_{j} R e_{j}}\left(e_{j} E_{i}, e_{j} E_{j}\right)$ by [6, Lemma 2.1]. Note that since the quivers of $R$ are trees, $R e_{i}$ and $e_{i} R$ are distributive $R$-modules for each $i=1, \cdots, n$ [3]. So by Lemma 3 and [7, Lemmas 4 and 5], ${ }_{e_{j} R e_{j}} e_{j} E_{i f_{i} S f_{i}} \cong \operatorname{Hom}_{R}\left(R e_{j}, E_{i}\right)$ is left and right uniserial, so since $f_{i} S f_{i}$ is also a division ring, $e_{j} E_{i}$ is both left and right one-dimensional or zero. Now since ${ }_{e_{j} R e_{j}} e_{j} E_{j}$ is also one-dimensional, it follows that ${ }_{f_{i} S f_{i}} f_{i} S f_{j}$ is zero or one-dimensional. Note also that $f_{i} S f_{j} \neq 0$ iff $e_{j} E_{i} \neq 0$ iff $e_{i} R e_{j} \neq 0$. Thus $R$ and $S$ are isomorphic factor rings of tic tac toe rings with the same quivers over isomorphic division rings $e_{i} R e_{i} \cong f_{i} S f_{i}$.

Regarding algebras of finite module type, we conclude with

REMARK 5. Let $R$ be an indecomposable hereditary artin algebra of finite module type which does not satisfy the hypotheses of Theorem 
2. Then according to Dlab and Ringel [4], [5] the quivers of $R$ or of its opposite ring are Dynkin diagrams of one of the types

$$
\begin{array}{ll}
B_{n}: v_{n} \longrightarrow \cdots-v_{2} \longleftarrow v_{1} & v_{n}^{\prime} \longrightarrow \cdots-v_{2}^{\prime} v_{1}^{\prime} \\
C_{n}: v_{1} \longleftarrow v_{2}-\cdots-v_{n} & v_{1}^{\prime} v_{2}^{\prime}-\cdots-v_{n}^{\prime} \\
F_{4}: v_{1} \longrightarrow v_{2} \longleftarrow v_{3} & v_{1}^{\prime} v_{2}^{\prime} v_{3}^{\prime}-v_{4}^{\prime} \\
G_{2}: v_{1} \longleftarrow v_{2} & v_{1}^{\prime} \rightleftarrows v_{2}^{\prime} .
\end{array}
$$

Using an argument similar to that in the proof of Theorem 2, one can show that if $R$ is an artinian ring with quivers of one of the above types, then $R$ is a factor ring of a generalized tic tac toe ring; that is, $R$ is isomorphic to a factor of a matrix ring with some of the entries from a division subring $C$ of a division ring $D$ and the other nonzero entries from $D$. (For example, if $R$ is hereditary with quivers

$$
\begin{aligned}
& \mathscr{Q}\left({ }_{R} R\right)=v_{3} \longrightarrow v_{1} \longleftrightarrow v_{2} \longleftrightarrow v_{4} \\
& \mathbb{Q}\left(R_{R}\right)=v_{3}^{\prime} \longleftrightarrow v_{1}^{\prime} \longrightarrow v_{2}^{\prime} \longrightarrow v_{4}^{\prime}
\end{aligned}
$$

then $R$ is isomorphic to a ring $T$ of matrices

$$
\left[\begin{array}{llll}
D & D & D & D \\
& C & 0 & C \\
& & D & 0 \\
& & & C
\end{array}\right]
$$

with $\operatorname{dim}\left(D_{C}\right)=2$.) To show this, assume that $R$ is basic and that $Q^{2}\left({ }_{R} R\right)$ is a tree. Arrange the right and left quivers of $R$ so that the multiple arrows point to the right. Let $v_{\alpha}^{\prime}$ be the vertex of $\mathbb{Q}\left(R_{R}\right)$ at the tails of the multiple arrows, and let $v_{\beta}^{\prime}$ be the vertex of $\mathscr{Q}\left(R_{R}\right)$ at the heads of the multiple arrows. Let $\mathscr{Q}_{\alpha}$ be the subquiver of $\mathscr{Q}\left({ }_{R} R\right)$ containing $v_{\alpha}$ and the arrows and vertices to the left of $v_{\alpha}$, and let $\mathbb{Q}_{\beta}$ be the subquiver of $\mathscr{Q}\left({ }_{R} R\right)$ containing $v_{\beta}$ and the arrows and vertices to the right of $v_{\beta}$. Notice that $\operatorname{dim}\left({ }_{e_{\alpha} R_{\alpha}} e_{\alpha} J e_{\beta}\right)=$ 1 since $\mathscr{Q}\left({ }_{R} R\right)$ is a tree. For $v_{p} \leftarrow v_{q}$ in $\mathscr{Q}\left({ }_{R} R\right)$, let $e_{p q}$ generate $e_{p} R e_{p} e_{p} J e_{q}$, and define $e_{j i}$ as before for $v_{i} \leqq v_{j}$. Define $\sigma_{\alpha j}$ for $v_{j} \in \mathbb{Q}_{\alpha}$ and $\sigma_{\beta j}$ for $v_{j} \in \mathbb{Q}_{\beta}$ as in the proof of Theorem 2. Let

$$
C^{\prime}=\left\{\sum_{v_{j} \in \mathscr{Q}_{\beta}} \sigma_{\beta j}(x) \mid x \in e_{\beta} R e_{\beta}\right\} .
$$

Let

$$
D=\left\{\sum_{v_{j} \in \mathscr{Q}_{\alpha}} \sigma_{\alpha j}(x) \mid x \in e_{\alpha} R e_{\alpha}\right\}
$$


Define $\theta: C^{\prime} \rightarrow D$ via $e_{\alpha \beta} e_{\beta} c^{\prime}=\theta\left(c^{\prime}\right) e_{\alpha} e_{\alpha \beta}$ for $c^{\prime} \in C^{\prime}$. Then $C=\operatorname{im} \theta \cong C^{\prime}$. Now let

$$
\begin{aligned}
T= & \left\{\llbracket d_{i j} \rrbracket \mid d_{i j} \in D, d_{i j}=0 \text { if } v_{i} \not v_{j},\right. \\
& \text { and } \left.d_{i j} \in C \text { if } v_{i} \in \mathbb{Q}_{\beta}\right\} .
\end{aligned}
$$

Then $T$ is a ring, since if $v_{k} \in \mathscr{Q}_{\alpha}$ and $v_{i} \in \mathscr{Q}_{\beta}$, then $v_{i} \not v_{k}$ (and hence $\left.d_{i k}=0\right)$. So in any nonzero product $d_{i j} d_{j k}$, we must have $v_{i} \leqq v_{j}$ and $v_{j} \leqq v_{k}$, giving $v_{i} \leqq v_{k}$, and thus either $v_{i} \in \mathscr{Q}_{\alpha}$, or both $v_{i}, v_{j} \in \mathbb{Q}_{\beta}$ and $d_{i j} d_{j k} \in C$. Now define

$$
\begin{array}{ll}
\Phi: & T \rightarrow R \quad \text { by } \\
\Phi: & \llbracket d_{i j} \rrbracket \longmapsto \\
v_{i} \leqq v_{j}, v_{i} \in \mathbb{Q}_{\beta} & \theta^{-1}\left(d_{i j}\right) e_{i j}+\sum_{v_{i} \leqq v_{j}, v_{i} \in \mathcal{C}_{\alpha}} d_{i j} e_{i j} .
\end{array}
$$

The map $\Phi$ is clearly additive and onto. To show that $\Phi$ preserves the multiplication, we need only add to the proof of Theorem 2 that for $d \in D$ and $c \in C$,

$$
d e_{\alpha \beta} \theta^{-1}(c) e_{\beta k}=d c e_{\alpha \beta} e_{\beta k}=d c e_{\alpha k},
$$

which is immediate by the definition of $\theta$.

ACKNowledgment. We wish to thank E. L. Green whose talks at The University of Iowa this fall were a principal source of inspiration for this work. Also we acknowledge that the first part of Lemma 1 was already known to him.

\section{REFERENCES}

1. F. W. Anderson and K. R. Fuller, Rings and Categories of Modules, SpringerVerlag, New York-Heidelberg-Berlin, 1974.

2. I. N. Bernstein, I. M. Gelfand, V. A. Ponomarev, Coxeter functors and a theorem of Gabriel, Uspehi Mat. Nauk., 28 (1973), 19-33.

3. V. P. Camillo, Distributive modules, J. Algebra, 36 (1975), 16-25.

4. V. Dlab and C. Ringel, On algebras of finite representation type, J. Algebra, 33 (1975), 306-394.

5. - Indecomposable representations of graphs and algebras, Mem. Amer. Math. Soc. No. 173, 6 (1976).

6. K. R. Fuller, On indecomposable injectives over artinian rings, Pacific J. Math., 29 (1969), 115-135.

7. — Rings of left invariant module type, Comm. in Algebra, 6 (1978), 153-167.

8. P. Gabriel, Unzerlegbare Darstellungen I, Manuscripta Math., 6 (1972), 71-103.

9. - Indecomposable Representations II, in "Symposia Mathematica" Vol. XI, Academic Press, New York-San Francisco-London, 1973, 81-104.

10. A. W. Goldie, Torsion-free modules and rings, J. Algebra, 3 (1964), 268-287.

11. R. Gordon and E. L. Green, Modules with cores and amalgamations of indecomposable modules, Mem. Amer. Math. Soc., No. 187, 10 (1977).

12. B. Mitchell, Theory of Categories, Academic Press, New York-London, 1965.

13. K. Morita, Duality of modules and its applications to the theory of rings with minimum condition, Sci. Rep. Tokyo Kyoiku Daigaku, 6 (1958), 85-142. 
14. I. Murase, On the structure of generalized uniserial rings I, Sci. Pap. Coll. Gen. Educ. Univ. Tokyo, 13 (1963), 1-22.

Received January 18, 1977.

THE UNIVERSITY OF IOWA

IOWA CITY, IA 52242 



\section{PACIFIC JOURNAL OF MATHEMATICS}

\section{EDITORS}

RICHARD ARENS (Managing Editor)

University of California

Los Angeles, CA 90024

Charles W. Curtis

University of Oregon

Eugene, OR 97403

C. C. MOORE

University of California

Berkeley, CA 94720

\section{J. DugundJI}

Department of Mathematics

University of Southern California

Los Angeles, CA 90007

R. FinN and J. Milgram

Stanford University

Stanford, CA 94305

\section{ASSOCIATE EDITORS}
E. F. BECKENBACH
B. H. NeumanN
F. WOLF
K. YosHIDA

\section{SUPPORTING INSTITUTIONS}

\author{
UNIVERSITY OF BRITISH COLUMBIA \\ CALIFORNIA INSTITUTE OF TECHNOLOGY \\ UNIVERSITY OF CALIFORNIA \\ MONTANA STATE UNIVERSITY \\ UNIVERSITY OF NEVADA, RENO \\ NEW MEXICO STATE UNIVERSITY \\ OREGON STATE UNIVERSITY \\ UNIVERSITY OF OREGON
}

\author{
UNIVERSITY OF SOUTHERN CALIFORNIA \\ STANFORD UNIVERSITY \\ UNIVERSITY OF HAWAII \\ UNIVERSITY OF TOKYO \\ UNIVERSITY OF UTAH \\ WASHINGTON STATE UNIVERSITY \\ UNIVERSITY OF WASHINGTON
}

The Supporting Institutions listed above contribute to the cost of publication of this Journal, but they are not owners or publishers and have no responsibility for its content or policies.

Mathematical papers intended for publication in the Pacific Journal of Mathematics should be in typed form or offset-reproduced, (not dittoed), double spaced with large margins. Please do not use built up fractions in the text of the manuscript. However, you may use them in the displayed equations. Underline Greek letters in red, German in green, and script in blue. The first paragraph or two must be capable of being used separately as a synopsis of the entire paper. Items of the bibliography should not be cited there unless absolutely necessary, in which case they must be identified by author and journal, rather than by item number. Manuscripts, in triplicate, may be sent to any one of the editors. Please classify according to the scheme of Math. Reviews, Index to Vol. 39. All other communications should be addressed to the managing editor, or Elaine Barth, University of California, Los Angeles, California, 90024.

50 reprints to each author are provided free for each article, only if page charges have been substantially paid. Additional copies may be obtained at cost in multiples of 50 .

The Pacific Journal of Mathematics is issued monthly as of January 1966. Regular subscription rate: $\$ 72.00$ a year (6 Vols., 12 issues). Special rate: $\$ 36.00$ a year to individual members of supporting institutions.

Subscriptions, orders for numbers issued in the last three calendar years, and changes of address should be sent to Pacific Journal of Mathematics, 103 Highland Boulevard, Berkeley, California, 94708. Older back numbers obtainable from Kraus Periodicals Co., Route 100, Millwood, NY 10546.

PUBLISHED BY PACIFIC JOURNAL OF MATHEMATICS, A NON-PROFIT CORPORATION

Printed at Kokusai Bunken Insatsusha (International Academic Printing Co., Ltd.). 8-8, 3-chome, Takadanobaba, Shinjuku-ku, Tokyo 160, Japan.

Copyright (C) 1978 by Pacific Journal of Mathematics Manufactured and first issued in Japan 


\section{Pacific Journal of Mathematics}

\section{Vol. 76, No. $2 \quad$ December, 1978}

Stephanie Brewster Brewer Taylor Alexander, Local and global convexity in complete Riemannian manifolds ...........................

Claudi Alsina i Català, On countable products and algebraic convexifications of probabilistic metric spaces ...............................

Joel David Berman and George Grätzer, Uniform representations of

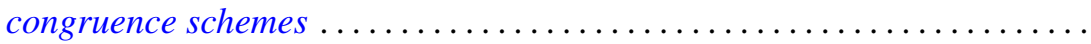

Ajit Kaur Chilana and Kenneth Allen Ross, Spectral synthesis in

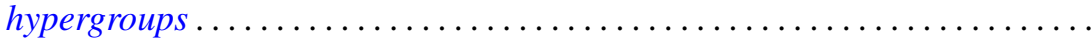

David Mordecai Cohen and Howard Leonard Resnikoff, Hermitian quadratic forms and Hermitian modular forms . .........................

Frank Rimi DeMeyer, Metabelian groups with an irreducible projective

representation of large degree .............................

Robert Ellis, The Furstenberg structure theorem .....................

Heinz W. Engl, Random fixed point theorems for multivalued mappings .......

William Andrew Ettling, On arc length sharpenings ..................

Kent Ralph Fuller and Joel K. Haack, Rings with quivers that are trees........

Kenneth R. Goodearl, Centers of regular self-injective rings ...............

John Gregory, Numerical algorithms for oscillation vectors of second order

differential equations including the Euler-Lagrange equation for

symmetric tridiagonal matrices.

Branko Grünbaum and Geoffrey Shephard, Isotoxal tilings

Myron Stanley Henry and Kenneth Leroy Wiggins, Applications of

approximation theory to differential equations with deviating

arguments

Mark Jungerman, The non-orientable genus of the n-cube .

Robert Richard Kallman, Only trivial Borel measures on $S_{\infty}$ are

quasi-invariant under automorphisms ................

Joyce Longman and Michael Rich, Scalar dependent algebras in the

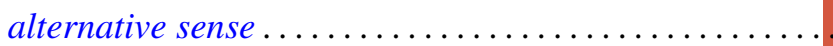

Richard A. Mollin, The Schur group of a field of characteristic zero ........ 471

David Pokrass, Some radical properties of rings with $(a, b, c)=(c, a, b) \ldots 479$

Margaret Shay and Paul Ruel Young, Characterizing the orders changed by

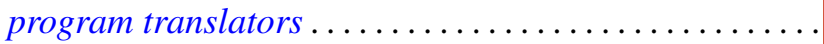

Jerrold Norman Siegel, On the structure of $B_{\infty}(F), F$ a stable space...

Surjeet Singh, (hnp)-rings over which every module admits a basic

submodule...

A. K. Snyder, Universal interpolating sets and the Nevanlinna-Pick property in

Banach spaces of functions...

Jeffrey D. Vaaler, On the metric theory of Diophantine approximation ... 